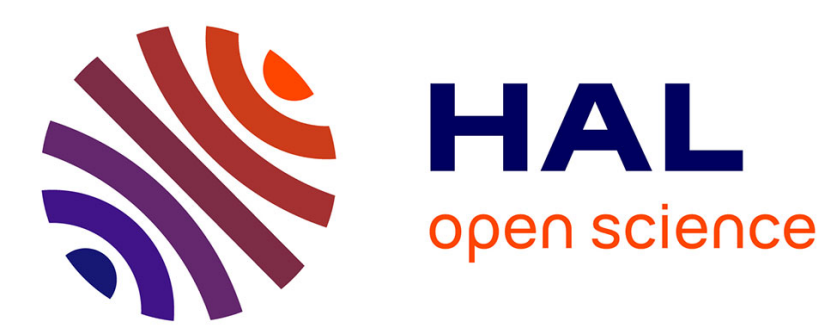

\title{
Osmotically induced deformation of capsid-like icosahedral vesicles
}

Gaelle Bealle, Jacques Jestin, David Carriere

\section{To cite this version:}

Gaelle Bealle, Jacques Jestin, David Carriere. Osmotically induced deformation of capsid-like icosahedral vesicles. Soft Matter, 2011, 7, pp.1084. 10.1039/C0SM00490A . cea-00870077

\section{HAL Id: cea-00870077 https://hal-cea.archives-ouvertes.fr/cea-00870077}

Submitted on 4 Oct 2013

HAL is a multi-disciplinary open access archive for the deposit and dissemination of scientific research documents, whether they are published or not. The documents may come from teaching and research institutions in France or abroad, or from public or private research centers.
L'archive ouverte pluridisciplinaire HAL, est destinée au dépôt et à la diffusion de documents scientifiques de niveau recherche, publiés ou non, émanant des établissements d'enseignement et de recherche français ou étrangers, des laboratoires publics ou privés. 


\title{
Osmotically induced deformation of capsid-like icosahedral vesicles $\dagger$
}

\author{
Gaëlle Béalle, ${ }^{a b}$ Jacques Jestin $^{c}$ and David Carrière $* a b$
}

\author{
Received 9th June 2010, Accepted 20th October 2010 \\ DOI: $10.1039 / \mathrm{c} 0 \mathrm{sm} 00490 \mathrm{a}$
}

\begin{abstract}
We report the osmotic deformation of micron-size catanionic vesicles with icosahedral symmetry (20 faces, 12 vertices) upon incubation in small solutes ( $\mathrm{NaCl}$, glucose). The vesicles remain icosahedral at low osmotic pressure gradients across the bilayer, or spherical for outwards gradients. Above a threshold value of inwards pressure, the icosahedra develop a buckling instability: a depression is initiated at one or two ridges, grows until one vertex snaps into the icosahedra, leading to full collapse of one half of the vesicle into the other. Despite large local inversions in curvature, no release of encapsulated solutes is observed before the residual volume reaches negligible small values. Thin shell models correctly capture the buckling patterns of icosahedra in the low deformation limit. Comparison of experimental results with Monte Carlo simulations provides a first estimate for the conditions of shell disruption, and suggests it is predominantly driven by curvature rather than two-dimensional stretching or compression. The relevance of these results for the mechanics of viral capsids and controlled release applications is discussed.
\end{abstract}

\section{Introduction}

Virus capsids are able to sustain the megapascal-range inwards and outwards osmotic gradients encountered in physiological conditions. $^{1,2}$ The mechanical conditions for the rupture of the capsid are of particular interest as they determine the amount of genetic materials confined in the shell, ${ }^{3}$ the resistance to osmotic shocks and for bacteriophages, the maximum ejection pressure of DNA in the host cell. ${ }^{2,4}$ Different models based on continuum mechanics of thin shells have been proposed to account for the spontaneous sphere-icosahedron transition, ${ }^{5}$ the deformations of icosahedra under different inwards and outwards mechanical loads, and finally their rupture.$^{6-8} \mathrm{Up}$ to now, these models have been tested experimentally on natural capsids submitted to indentation by atomic force microscopy, i.e. under anisotropic loading only., ${ }^{3-12}$ By contrast, the bucklingt figures of icosahedral shells submitted to isotropic pressure, which is more relevant biologically, have still to be elucidated, as imaging at the nanometre scale without perturbation of the osmotic pressure remains an experimental challenge.

Here, we mimic the osmotic deformation of virus using vesicles with icosahedral symmetry and sizes in the micron range. The deformation of such vesicles can be followed directly by confocal

${ }^{a}$ CEA, IRAMIS, UMR 3299 SIS2M, LIONS (Laboratoire Interdisciplinaire sur l'Organisation Nanométrique et Supramoléculaire), F-91191 Gif-sur-Yvette cedex, France. E-mail: david.carriere@cea.fr; Fax: +33(0)16908 66 40; Tel: +33(0)169085489

${ }^{b}$ CNRS, IRAMIS, UMR 3299 SIS2M, LIONS (Laboratoire Interdisciplinaire sur l'Organisation Nanométrique et Supramoléculaire), F-91191 Gif-sur-Yvette cedex, France

${ }^{c} C E A$, IRAMIS, LLB (CNRS, UMR 12) (Laboratoire Léon Brillouin), F-91191 Gif-sur-Yvette cedex, France

$\dagger$ Electronic supplementary information (ESI) available: Slow sphere-to-icosahedron transition upon ageing, parameters for the SANS fitting, and destruction of vesicles upon encapsulation of high glucose concentrations. See DOI: 10.1039/c0sm00490a

\$ In this paper, we use the term "buckling" exclusively to describe the inwards buckling of icosahedra. In literature, "buckling" sometimes also refers to the sphere-to-icosahedron transition. microscopy. Different mixtures of surfactants of opposite charge, also referred to as "catanionic mixtures", ${ }^{13-15}$ have been reported to form icosahedral vesicles (regular polyhedra with 12 vertices and 20 faces). ${ }^{16-19}$ In addition, for this study, we use catanionic vesicles with a weak permeability to small solutes. We show that upon incubation in solutions of $\mathrm{NaCl}$ or glucose, a buckling instability develops leading to characteristic facetted concave polyhedra. We show that the theory correctly predicts the deformation of these icosahedra under isotropic pressure and allows extracting from experimental data an estimate of the conditions at which bilayer rupture occurs.

\section{Materials and methods}

\section{Vesicle preparation and osmotic stress}

In a typical preparation, $31.3 \mathrm{mg}$ of cetyltrimethylammonium chloride (Acros), $41.8 \mathrm{mg}$ of myristic acid (Fluka, recrystallized twice from hot acetonitrile) and $7.497 \mathrm{~g}$ of water (MilliQ), or optionally of a $10 \mu \mathrm{M}$ Rhodamine 6G (Invitrogen) solution in water, are stirred at $50{ }^{\circ} \mathrm{C}$ during 3 days. The white birefringent solution is then diluted 3 -fold with water at $50{ }^{\circ} \mathrm{C}$ and stirred at $50{ }^{\circ} \mathrm{C}$ for 30 minutes. Five millilitres of the mixture are then dialyzed at room temperature against $500 \mathrm{~mL}$ of MilliQ-grade water (Float-a-Lyzer, regenerated cellulose, $8-10 \mathrm{kDa}$ ). The dialysis bath is changed every hour during the first 8 hours, and then every 12 hours during the next 4 days. ${ }^{20}$

For osmotic stress experiments, the solution of vesicles is diluted 2-fold with a solution of $\mathrm{NaCl}$ (Riedel-de Haën) or a freshly prepared solution of glucose (Sigma).

\section{Confocal microscopy}

Observations have been performed after staining with Oregon Green 488 (Invitrogen, 5\% weight of saturated solution), or directly on vesicles encapsulating Rhodamine $6 \mathrm{G}$ prepared as described above. Oregon Green $\left(\lambda_{\text {exc }}=488 \mathrm{~nm}\right)$ was observed in the $505-525 \mathrm{~nm}$ range and Rhodamine $6 \mathrm{G}\left(\lambda_{\mathrm{exc}}=543 \mathrm{~nm}\right)$ was 
observed above $560 \mathrm{~nm}$ using an inverse microscope (Olympus, IX 81) coupled to an FV 1000 confocal head (Olympus).

\section{Small angle neutron scattering}

Vesicles for SANS experiments were prepared with deuterated myristic acid (Sigma) and $\mathrm{H}_{2} \mathrm{O}$. After four days of dialysis, the sample was dialyzed at least three times against $\mathrm{D}_{2} \mathrm{O}$ in a $1 / 1$ sample/bath volume ratio during 3 hours, and then twice 12 hours in a $1 / 100$ ratio. Solutions of $\mathrm{NaCl}$ or glucose prepared in $\mathrm{D}_{2} \mathrm{O}$ were then added to the vesicles.

Measurements were performed at the Laboratoire Léon Brillouin (LLB) on the SANS two dimensional detector spectrometer PAXE. One configuration was used at wavelength $5 \AA$ and a sample-to-detector distance of $1 \mathrm{~m}$ with a collimation distance of $1.00 \mathrm{~m}$ corresponding to a total wave vector range of 0.015 to $0.20 \AA^{-1}$. Solutions were measured in $2 \mathrm{~mm}$-thick Hellma cells. Data processing was performed with a homemade program following standard procedures. To get the cross-section per volume in absolute units $\left(\mathrm{cm}^{-1}\right)$, the incoherent scattering crosssection of $\mathrm{H}_{2} \mathrm{O}$ was used as a calibration. It was estimated from the measurement of the attenuator strength and of the direct beam with the same attenuator. The incoherent scattering background, due to protons of the solvent, was subtracted using a blank sample as a function of the concentration of the scattered species.

The intensity scattered by as-prepared vesicles was fitted using the non-linear least square method with a two-layer heterogeneous plane symmetric along the midplane: ${ }^{21}$

$$
I_{1}(q)=\frac{8 \pi \Sigma}{q^{4}}\left(\sum_{i=1}^{2}\left(\rho_{i}-\rho_{i-1}\right) \sin \left(q d_{i}\right)\right)^{2}
$$

where $\Sigma$ is the specific area of the bilayers, $\rho_{0}$ the scattering length density of $\mathrm{D}_{2} \mathrm{O}, \rho_{i}$ the scattering length density of the $i^{\text {th }}$ layer and $d_{i}$ the distance between the midplane of the bilayer and the boundary between the layers $i$ and $i-1$. The mean scattering length per surfactant $\left(\rho_{1}\left(d_{1}-d_{2}\right)+\rho_{2} d_{2}\right) a_{0}$ was constrained by the mean chemical composition of the bilayer $\left(181 \times 10^{-15} \mathrm{~m}\right.$ at $f=0.66)$, using a surface per surfactant $a_{0}$ of $20.5 \AA^{2}$ as measured previously. ${ }^{22,23}$ The intensity scattered by pairs of bilayers separated by the distance $D$ is given by:

$$
I_{2}(q)=2 \cos ^{2}\left(q\left(\frac{D}{2}+d_{1}\right)\right) I_{1}(q)
$$

The SANS patterns of the vesicles under osmotic stress were therefore adjusted with a non-linear least square fit using:

$$
I(q)=\frac{8 \pi \Sigma}{q^{4}}\left(\sum_{i=1}^{2}\left(\rho_{i}-\rho_{i-1}\right) \sin \left(q d_{i}\right)\right)^{2}\left(1+\phi \cos \left(D+2 d_{1}\right)\right)
$$

where $\phi$ is the fraction of bilayers associated in pairs. Only $\phi$, $D$ and $\Sigma$ were used as free parameters for the fit, all other parameters were kept equal to the values determined on asprepared vesicles except for $\rho_{0}$ that was corrected for the scattering length density of the solute.

\section{Monte Carlo simulations}

We have performed equilibration at $T=298 \mathrm{~K}$ of surfaces described by a regular triangulation with $10 h^{2}+2$ particles, 12 of them showing a 5-fold valency (the vertices of the icosahedron), the others a 6-fold valency (the mapping of rest of the icosahedron). This corresponds to the $(h, k=0)$ icosahedral polygonisation in the Caspar-Klug construction. ${ }^{24}$ We used the Hamiltonian already proposed by Siber, ${ }^{6}$ or alternatively by Buenemann and Lenz, ${ }^{12}$ with no noticeable difference:

$$
H_{s}=\frac{\sqrt{3}}{4} Y \sum_{i, j}\left(\left|\vec{r}_{i}-\vec{r}_{j}\right|-a\right)^{2}
$$

for the stretching contribution, where the sum runs over neighboring particles of indices $(i, j)$ of position $\vec{r}, Y$ is the 2D Young's Modulus, and $a$ the equilibrium distance of two neighbors, and

$$
H_{\mathrm{b}}=\frac{1}{\sqrt{3}} \kappa \sum_{I, J}\left(\vec{n}_{I}-\vec{n}_{J}\right)^{2}
$$

for the bending contribution, where the sum runs over neighboring triangles of indices $(I, J)$ of normal vectors $\vec{n}$, and $\kappa$ the bending modulus.

The energy was first minimized using a standard simulated annealing procedure using a power law decay and $T=298 \mathrm{~K}$ as the final temperature. ${ }^{25}$ Prior to cycling at each new temperature step, the amplitude of the random variations in vertices positions was set to achieve an initial approval ratio of $0.5 \pm 10 \%$. Each cycle during annealing consisted of 20 random trials per particle, with at least 1002 particles $(h \geq 10)$. Cycling at given temperature was interrupted when the energy statistical variations over the last 100 cycles followed a normal distribution. Once equilibrium at $T=298 \mathrm{~K}$ was reached, statistical accumulation of strain and curvature was performed over 100 Monte Carlo cycles.

Concave icosahedral vesicles with a single vertex buckled in could be equilibrated without setting any external pressure nor volume constraint, and did not collapse or swell back, even with annealing from $T=298000 \mathrm{~K}$. In addition, we have checked the consistency of our simulations with results reported previously by Buenemann and Lenz, ${ }^{12}$ and Siber and Podgornik. ${ }^{8}$

The strain of buckled vesicles after equilibration is:

$$
\varepsilon=\frac{d_{i j}-d_{i j}^{0}}{d_{i j}^{0}}
$$

where $d_{i j}$ and $d_{i j}{ }^{0}$ are the mean distances between neighboring particles $i$ and $j$ in the buckled vesicle and unbuckled vesicles, respectively. The mean curvature at particle $i$ was calculated by identifying eqn (5) to the continuum limit of the bending Hamiltonian. $^{26}$

\section{Results and discussion}

\section{Sphere-to-icosahedron transition}

The vesicles under consideration are obtained from a two-step preparation: ${ }^{20}$ (i) co-solubilization of myristic acid $\left(\mathrm{C}_{13} \mathrm{COOH}\right)$ and cetyltrimethylammonium chloride $\left(\mathrm{C}_{16} \mathrm{TA}^{+} \mathrm{Cl}^{-}\right)$in a $2: 1$ ratio, which yields elongated vesicles and releases $\mathrm{HCl}$ as anionic and cationic surfactants from the bilayer, (ii) dialysis which eliminates exclusively the ions outside the vesicles, while about $2 \mathrm{mM} \mathrm{HCl}$ remain encapsulated inside. ${ }^{22}$ Confocal microscopy images of the as-prepared vesicles stained by an anionic dye 
(Oregon Green, 5\% w/w saturated solution) or equivalently by encapsulation of a cationic dye (Rhodamine $6 \mathrm{G}, 10 \mu \mathrm{M}$ ) demonstrate that their shape is spherical (Fig. 1a). As demonstrated previously, the mixed surfactant bilayer is in the solid state ("gel-phase"). ${ }^{20,22}$

After incubation in $\mathrm{NaCl}(<1$ to $2 \mathrm{mM})$ or glucose $(<2$ to $3 \mathrm{mM}$ ) for 24 hours, the spherical vesicles develop kinks and facets which become more pronounced as the solute concentration increases within this range. At concentrations close to $1 \mathrm{mM}$ $\mathrm{NaCl}$ (or equivalently $2 \mathrm{mM}$ glucose), well-defined patterns with 5-fold and 6-fold symmetries are predominantly observed (Fig. 1b-d), which are interpreted as icosahedral vesicles with different orientations with respect to the focal plane. This icosahedral shape is assigned to the form adopted by the vesicles at zero osmotic gradient across the bilayer, i.e. when the added solute compensates for the $\mathrm{HCl}$ encapsulated into the asprepared vesicles. Consistently with our interpretation, the vesicles reported by Dubois et al., ${ }^{19}$ which only differ by replacement of $\mathrm{Cl}^{-}$with $\mathrm{OH}^{-}$, are both porous and spontaneously icosahedral, whereas pristine spherical vesicles prepared from $\mathrm{CTA}^{+} \mathrm{Cl}^{-}$slowly (months) develop facets and vertices as a result of $\mathrm{HCl}$ leakage across the bilayer (ESI $\dagger$ ).

The icosahedral shape in our system is assigned to the combination of mechanical and topological requirements: (i) a high bilayer stretching energy as compared to the bending energy favours a sphere-to-icosahedra transition when the Foppl-Von-Kàrmàn parameter $\gamma=Y R^{2} / \kappa$ is larger than a critical value in the 500-1000 range, where $Y$ is the two-dimensional Young's modulus of the bilayer, $R$ the radius of the vesicle, and $\kappa$ the bending modulus ${ }^{5}$ and (ii) a spherical geometry is topologically incompatible with a bilayer showing a local $n$-fold geometry, which will favour the creation of $2 n$ defects (leading here to twelve vertices for a hexagonal local order). ${ }^{27,28}$ The latter requirement probably prevents giant phospholipid vesicles to form icosahedra although the mechanical constraints are usually met (e.g. $Y \approx 0.1 \mathrm{~N} \mathrm{~m}^{-1}, \kappa \approx 10^{-18} \mathrm{~J}$ for DMPC and $\left.\mathrm{DPPC}^{29-31}\right)$. Alternative mechanisms for the formation of icosahedra from vesicles have been proposed in literature, including the formation of supernetworks of alternate charged headgroups ${ }^{32}$ or the lateral phase segregation of charge excess at vertices of icosahedra. ${ }^{33}$ However, it has been demonstrated experimentally that neither mechanism is applicable in this

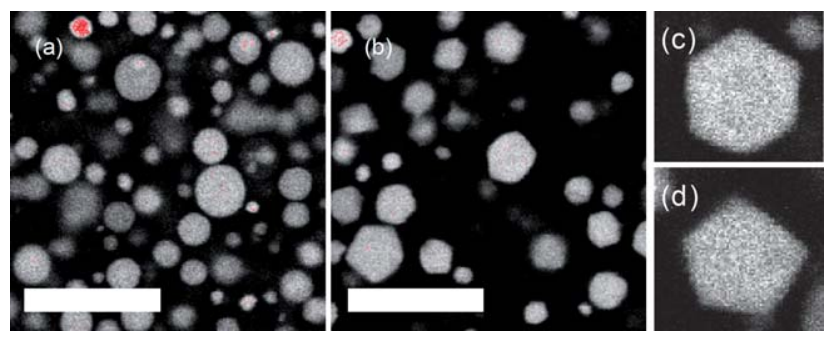

Fig. 1 Spherical and icosahedral vesicles below the inwards buckling transition. Confocal microscopy images (Rhodamine 6G channel) of (a) as-prepared vesicles with encapsulated Rhodamine $6 \mathrm{G}(\mathrm{b})$ the same after incubation in $0.5 \mathrm{mM} \mathrm{NaCl}$ (c) and (d) selected magnifications. The bar is $20 \mu \mathrm{m}$. system, using neutron diffraction ${ }^{34}$ and calorimetric studies, ${ }^{35}$ respectively.

\section{Buckling of icosahedra}

The icosahedral $\mathrm{CTA}^{+} \mathrm{Cl}^{-} / \mathrm{C}_{13} \mathrm{COOH}$ vesicles incubated in $1 \mathrm{mM}$ $\mathrm{NaCl}$ or $2 \mathrm{mM}$ glucose do not show significant evolution in shape nor leakage of Rhodamine 6G (if initially encapsulated) over several weeks. By contrast, higher solute concentrations lead to drastic changes in shapes (Fig. 2 and 3). After the spheres to icosahedra transition, facetted concave polygons with welldefined angles and edges appear (Fig. 2a). In particular, several characteristic patterns are assigned to two different buckling modes: (i) hexagons and pentagons with the middle of one edge pointing to the centre, distorted pentagons with two right angles and pairs of parallel diamond-like patterns (Fig. 3a-c) are assigned to inwards buckling of the middle of one ridge of the icosahedron, (ii) similar patterns with two apices (Fig. 3d-f), assigned to inwards buckling of two ridges sharing a common vertex.

At longer times the vesicles further collapse (Fig. 2b), leading to patterns where the inwardly oriented apices are more acute and closer to the opposite wall of the vesicle. Additional characteristic patterns are also observed (Fig. 3g-j), which consist of polygons with a darker central area, and of concave polygons with shorter inner separations. These patterns observed at longer times only are not compatible with the above-mentioned buckling from ridges, but are assigned to icosahedra with a single vertex buckled inwardly and located around the geometrical centre of the initial vesicle.
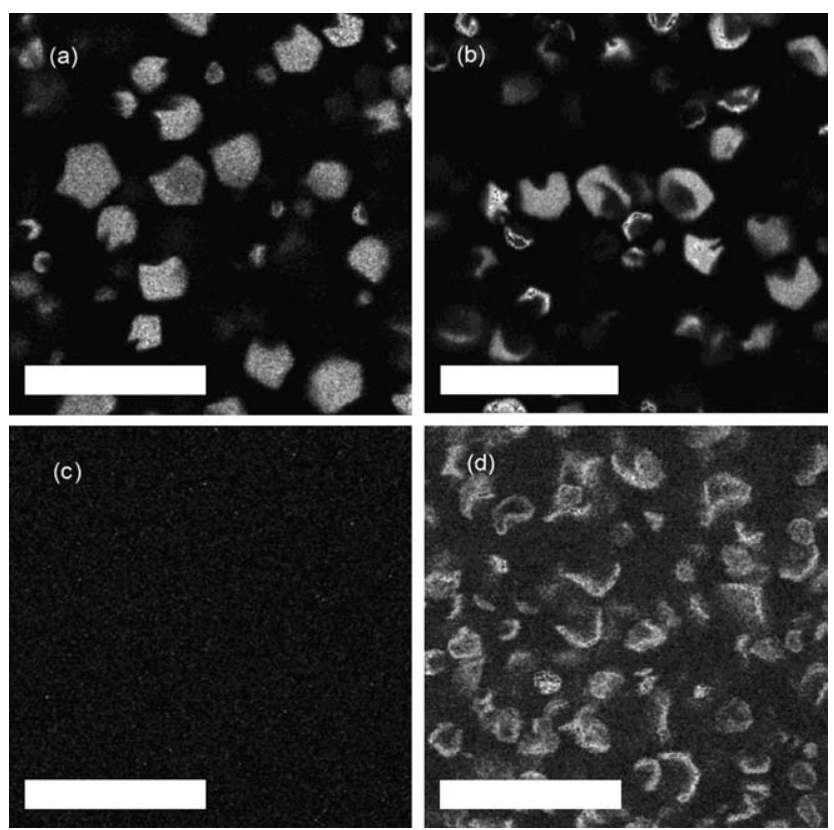

Fig. 2 Vesicles above the inwards buckling transition. Confocal microscopy images of vesicles with encapsulated Rhodamine 6G incubated in $20 \mathrm{mM}$ glucose after (a) 85 minutes, Rhodamine 6G channel (b) 183 minutes, Rhodamine 6G channel (c) 24 hours, Rhodamine 6G channel (d) 24 hours, after addition of Oregon Green, observation of the Oregon Green channel. The bars are $20 \mu \mathrm{m}$. 


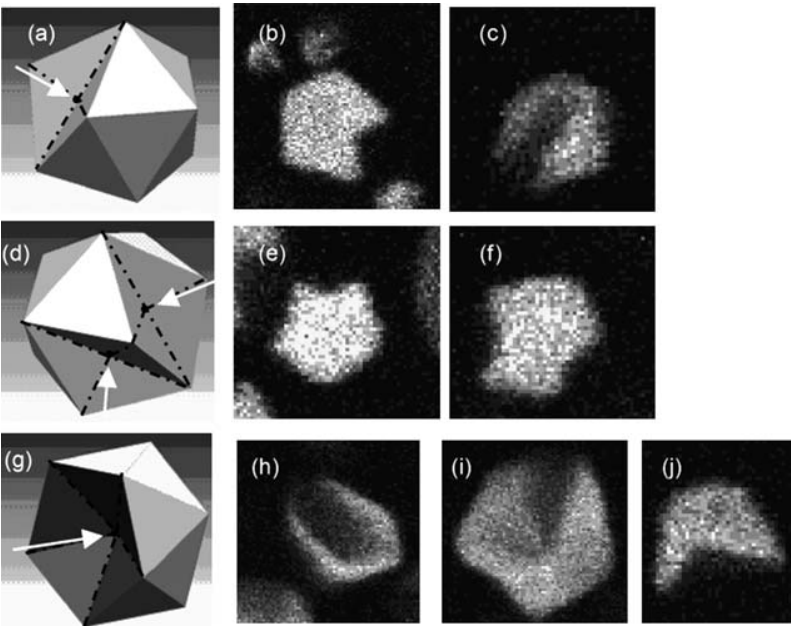

Fig. 3 Details of the buckling patterns. Sketches and corresponding selected confocal images of $(a-c)$ icosahedra with a single ridge buckled in $(d-f)$ icosahedra with two ridges buckled in $(g-j)$ icosahedra with a vertex buckled in. The arrows indicate the directions of buckling.

After one to three days (depending on solute concentration), no fluorescence contrast is observed anymore between the interior of vesicles initially loaded with the cationic Rhodamine $6 \mathrm{G}$ and the outer solution (Fig. 2c). This indicates that the vesicles have become permeable to the dye molecules. However, staining with an anionic dye (Oregon Green 488) still reveals the vesicles by accumulation at the positively charged bilayers. ${ }^{20}$ Coexistence between polygons and thin lines with one or two kinks and showing axial symmetry is observed (Fig. 2d). These patterns are assigned to complete buckling of icosahedra, i.e. one half of the vesicle snapped into the other, and a separation between bilayers below the resolution of confocal microscopy. In this regime, Small Angle Neutron Scattering (SANS) allows determining the mean distance between bilayers. As the concentration of added solute increases, the SANS patterns after three days incubation evolve from the signature characteristic of independent bilayers (micron-size swollen vesicles), to a curve with two and more peaks at smaller angles (Fig. 4a). Those peaks are assigned to the bilayer-bilayer correlations within the buckled vesicles, as quantitatively supported by modelling the scattering pattern by pairs of bilayers (Fig. 4a lines, Experimental and ESI $\dagger$ ). The mean bilayer separation inside the buckled icosahedra is thus found to decrease down to the nanometre range (Fig. 4b). Below $100 \mathrm{mM}$, the bilayer separations at given solute osmolarity (i.e. twice the concentration for $\mathrm{NaCl}$ ) are identical within $4 \%$. The specific area of bilayers (surface of bilayers per volume unit) remains unchanged upon incubation within experimental accuracy, which indicates that no bilayer dissolution occurs. This quantitatively supports that the collapse of the icosahedra is driven osmotically, at least until the bilayer separation reaches $10 \mathrm{~nm}$.

\section{Comparison with theory}

We observe that the transition from icosahedra to fully collapsed icosahedra is a buckling instability: below the critical concentration of solute, the icosahedra do not evolve. Above this
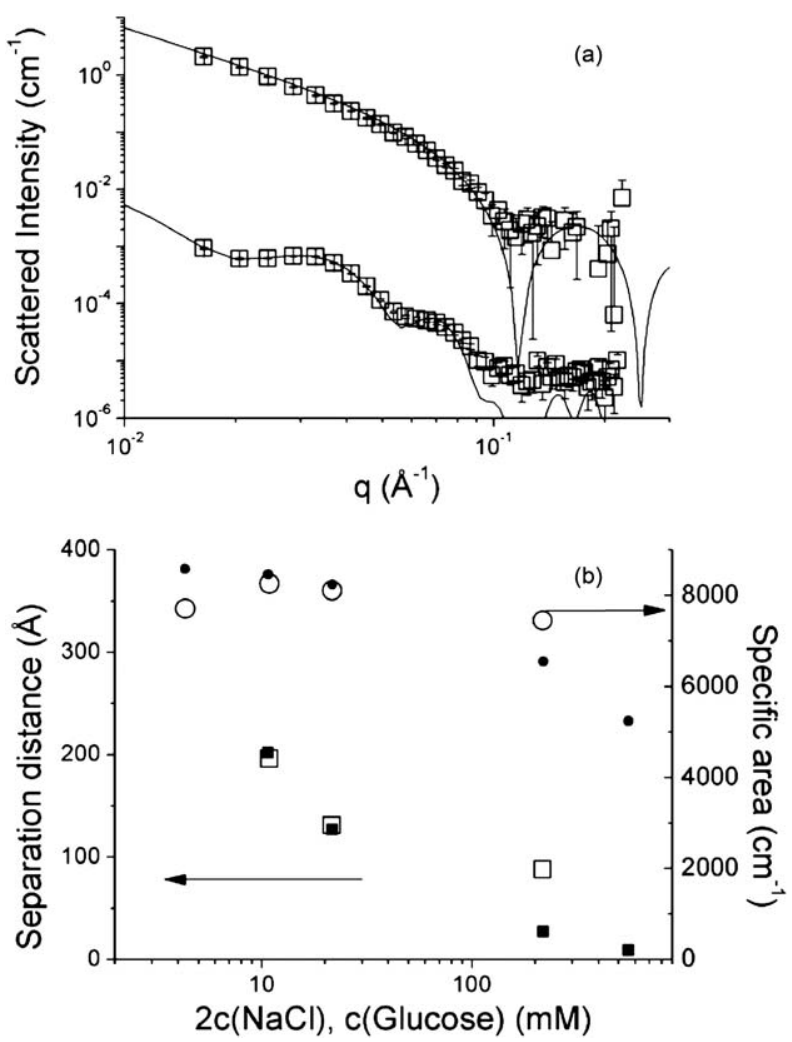

Fig. 4 Bilayer concentration and bilayer-bilayer separation. (a) SANS patterns of as-prepared vesicles (top) and after incubation in $20 \mathrm{mM}$ glucose (bottom, divided by $10^{3}$ for clarity). Squares: experimental data. Lines: fit to eqn (1) (top) and (3) (bottom) (b) bilayer-bilayer separations (squares) and total bilayer concentrations (circles) as determined by SANS after incubation in $\mathrm{NaCl}$ (open symbols) and glucose (filled symbols).

concentration, complete buckling always occurs, the various intermediate stages described above are only transient if the pressure (solute concentration) is maintained. Comparison of our experimental observations with current predictions on the buckling of icosahedral shells requires in a preliminary step to evaluate the mechanical parameters of the bilayer. Following the approach of Buenemann and Lenz, ${ }^{12}$ we extract a Foppl-VonKàrmàn parameter $\gamma \approx 6 \times 10^{4}$, with $Y \approx 0.6 \mathrm{~N} \mathrm{~m}^{-1}$ and $\kappa \approx$ $10^{-17} \mathrm{~J}$ by comparing the spring constants at vertices and facets as determined by atomic force microscopy by Delorme et al. ${ }^{36}$ $\left(50 \mathrm{mN} \mathrm{m}^{-1}\right.$ and $5 \mathrm{mN} \mathrm{m}^{-1}$ respectively). As already pointed out, the bending modulus for these catanionic systems is significantly higher than that reported for phospholipid bilayers in the gel phase (vide supra), and its value is confirmed independently by the onset of bilayer disruption under internal pressure: the density in vesicles recovered after preparation drastically decreases above encapsulation of $40 \mathrm{mOsm} \mathrm{NaCl}$ or glucose (ESI $\dagger$ ) due to destruction upon dialysis. This corresponds to bilayer disruption at $100 \mathrm{kPa}$, which scales correctly with the value predicted by Buenemann and Lenz, ${ }^{12}$ i.e. $p_{\text {rupture }} \approx 0.1 \times$ $Y / R=60 \mathrm{kPa}$, with little dependency on $\gamma$.

With $\gamma$ in the $10^{4}$ to $10^{5}$ range, the onset of inwards buckling as predicted by Siber and Podgornik ${ }^{8}$ is expected at $p_{\text {buckling }} \approx 100 \kappa /$ $R^{3}=1 \mathrm{kPa}$, i.e. at osmolarity gradients above $0.4 \mathrm{mOsm}$. It scales properly with our observations within experimental accuracy, as 
the onset of inwards buckling is observed at less than $1 \mathrm{mOsm}$ above the sphere-to-icosahedra transition. In addition, the slow kinetics allows direct observation of the buckling patterns and comparison with theory. Under isotropic pressure, buckling is expected to start preferentially at faces or ridges, while vertices keep pointing outwards. ${ }^{8,37}$ This is consistent with our observations of the early stages of deformations where only one or two ridges buckle inwards before vertices snap in (Fig. 2 and 3).

Despite this local inversion in bilayer curvature, no release of encapsulated species is observed in these first steps, even several hours after inversion (Fig. 2a and b). Consistently, icosahedra with one vertex buckled inwards swell back to spherical vesicles upon dialysis, due to the $\mathrm{HCl}$ naturally present. By contrast, leakage of $\mathrm{HCl}$ and of Rhodamine $6 \mathrm{G}$ is observed from vesicles in the late stages of buckling, i.e. which appear flat under confocal microscopy (Fig. 2c). Consistently, such vesicles do not swell back upon dialysis. This leakage coincides with bilayer separations of a few tens of nanometres becoming detectable (Fig. 4b), and therefore suggests that rupture of such gel-phase bilayer occurs at locations where the radius of curvature becomes comparable to the thickness $(\sim 4 \mathrm{~nm}$, ref. 23$)$.
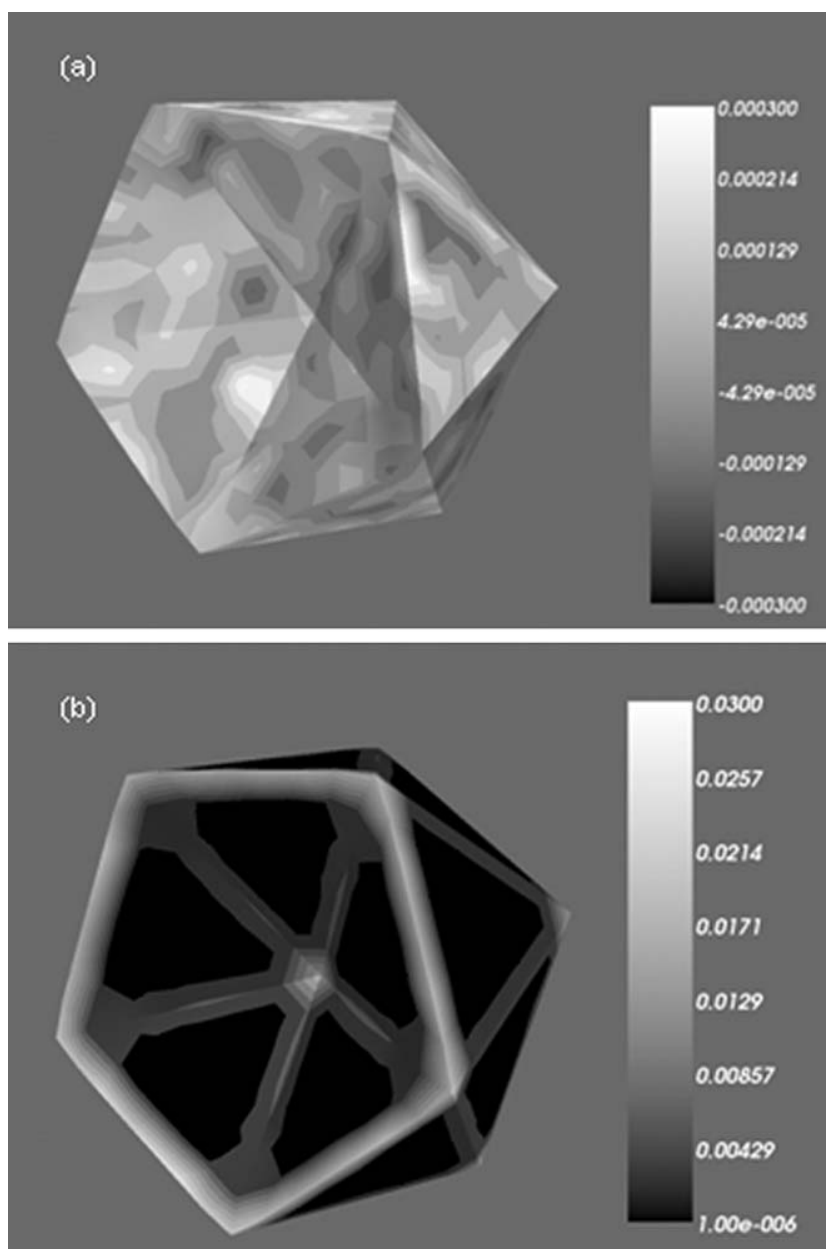

Fig. 5 Monte Carlo snapshot of the equilibrium shape of an icosahedral vesicle with a single vertex snapped in. (a) Contour plot of the strain calculated from 100 accumulations at equilibrium, standard deviation is $5 \times 10^{-4}$. (b) Contour plot of the curvature in $\mathrm{nm}^{-1}$, standard deviation is $10^{-4} \mathrm{~nm}^{-1}$.
Our results therefore demonstrate that mechanical models based on discretization of the bilayer capture qualitatively the deformation modes of icosahedral vesicles at the onset of inwards buckling under isotropic pressure. In addition, we have performed similar simulations, using a simulated annealing procedure followed by a Metropolis Monte Carlo algorithm to calculate the mean local strain and curvature in bilayers of deformed vesicles at $T=298 \mathrm{~K}$ (Materials and methods). Using the mechanical moduli determined above, it shows that in vesicles with a single vertex buckled into the icosahedra (Fig. 5), the deformation by stretching or compression in the facets is negligible: the maximum absolute value of the strain is about $\varepsilon=3 \times 10^{-4} \pm 5 \times 10^{-4}$ (Fig. 5a).

By contrast, the deformation by bending is very significant: the radius of curvature is $37 \mathrm{~nm} \pm 0.1 \mathrm{~nm}$ at the pentagonal rim where the bilayer becomes concave (Fig. 5b), against $350 \mathrm{~nm}$ before buckling. As the bilayer thickness is around $4 \mathrm{~nm}$, the area of the outer side of the bilayer is therefore locally $10 \%$ higher than the area of the inner side. However, as the bilayers are poorly compressible, such strong bending must involve molecular rearrangement in the bilayers, e.g. flip-flop. It is not clear from our experiments whether this curvature change occurs at constant half-bilayer composition, or if it is coupled to a change in local composition and/or surface charge density, as proposed by other authors. ${ }^{16,33}$ Combining the different results from experiments and modeling presented here, we estimate that bilayer rupture, as monitored by the release of encapsulated species, is probably driven by curvature, not stretching, and occurs as the local radius of curvature is below $40 \mathrm{~nm}$ (from Monte Carlo simulations) and roughly above $5 \mathrm{~nm}$ (from SANS). A detailed and time-resolved investigation of the rupture mechanism is currently carried out to refine these observations, in particular how curvature inversion at one vertex can occur without irreversible pore opening.

\section{Conclusions}

The onset of the buckling instability and the sequence of concave shapes observed upon incubation of catanionic icosahedral vesicles impermeable to small solutes are properly described by thin shell theory. Our observations show that in these systems where the non-dimensional Foppl-Von-Kàrmàn ratio is very large, bilayer rupture upon osmotic stress must predominantly occur when the radius of curvature becomes commensurate with the thickness. This contrasts with previous results obtained by squeezing vesicles between an AFM tip and a substrate, where the experimental geometry accounts for rupture by compression. However, some caution and more detailed experiments are needed before extrapolating these results to usual viruses, but they may be more safely transposed to mimivirus which have diameters as large as $800 \mathrm{~nm}^{38}$ and are therefore more suitable to analysis with thin shell theory. In addition, this system suggests possible strategies for controlled delivery applications: due to the specific mechanical properties of these vesicles, the buckling instability leads to full collapse of the shell and complete release of its contents even at moderate hypertonic conditions, adjustable by encapsulation of passive solutes to displace the osmotic threshold. 


\section{Acknowledgements}

The authors acknowledge Luc Belloni, Jean Daillant and Olivier Spalla for fruitful discussions, and Patrick Haltebourg for assistance with the Catia software.

\section{Notes and references}

1 T. F. Anderson, C. Rappaport and N. A. Muscatine, Ann. Inst. Pasteur (Paris), 1953, 84, 5-14.

2 D. E. Smith, S. J. Tans, S. B. Smith, S. Grimes, D. L. Anderson and C. Bustamante, Nature, 2001, 413, 748-752.

3 I. Ivanovska, G. Wuite, B. Jonsson and A. Evilevitch, Proc. Natl. Acad. Sci. U. S. A., 2007, 104, 9603-9608.

4 A. Evilevitch, L. Lavelle, C. M. Knobler, E. Raspaud and W. M. Gelbart, Proc. Natl. Acad. Sci. U. S. A., 2003, 100, 92929295.

5 J. Lidmar, L. Mirny and D. R. Nelson, Phys. Rev. E: Stat., Nonlinear, Soft Matter Phys., 2003, 68, 051910.

6 A. Siber, Phys. Rev. E: Stat., Nonlinear, Soft Matter Phys., 2006, 73, 061915.

7 T. T. Nguyen, R. F. Bruinsma and W. M. Gelbart, Phys. Rev. E: Stat., Nonlinear, Soft Matter Phys., 2005, 72, 051923.

8 A. Siber and R. Podgornik, Phys. Rev. E: Stat., Nonlinear, Soft Matter Phys., 2009, 79, 011919.

9 W. S. Klug, R. F. Bruinsma, J. P. Michel, C. M. Knobler, I. L. Ivanovska, C. F. Schmidt and G. J. L. Wuite, Phys. Rev. Lett., 2006, 97, 228101.

10 J. P. Michel, I. L. Ivanovska, M. M. Gibbons, W. S. Klug, C. M. Knobler, G. J. L. Wuite and C. F. Schmidt, Proc. Natl. Acad. Sci. U. S. A., 2006, 103, 6184-6189.

11 I. L. Ivanovska, P. J. de Pablo, B. Ibarra, G. Sgalari, F. C. MacKintosh, J. L. Carrascosa, C. F. Schmidt and G. J. L. Wuite, Proc. Natl. Acad. Sci. U. S. A., 2004, 101, 7600-7605.

12 M. Buenemann and P. Lenz, Proc. Natl. Acad. Sci. U. S. A., 2007, 104, 9925-9930.

13 P. Jokela, B. Jonsson and A. Khan, J. Phys. Chem., 1987, 91, 32913298.

14 E. W. Kaler, K. A. Murthy, B. E. Rodriguez and J. A. N. Zasadzinski, Science, 1989, 245, 1371-1374.

15 T. Zemb, D. Carriere, K. Glinel, M. Hartman, A. Meister, C. Vautrin, N. Delorme, A. Fery and M. Dubois, Colloids Surf., A, 2007, 303, $37-45$.
16 A. Gonzalez-Perez, M. Schmutz, G. Waton, M. J. Romero and M. P. Krafft, J. Am. Chem. Soc., 2007, 129, 756-757.

17 M. A. Greenfield, L. C. Palmer, G. Vernizzi, M. O. de la Cruz and S. I. Stupp, J. Am. Chem. Soc., 2009, 131, 12030-12031.

18 F. E. Antunes, R. O. Brito, E. F. Marques, B. Lindman and M. Miguel, J. Phys. Chem. B, 2007, 111, 116-123.

19 M. Dubois, B. Deme, T. Gulik-Krzywicki, J. C. Dedieu, C. Vautrin, S. Desert, E. Perez and T. Zemb, Nature, 2001, 411, 672-675.

20 Y. Michina, D. Carrière, C. Mariet, M. Moskura, P. Berthault, L. Belloni and T. Zemb, Langmuir, 2009, 25, 698-706.

21 Neutron, X-Rays and Light Scattering, ed. P. Lindner and T. Zemb, North-Holland, 1991.

22 D. Kopetzki, Y. Michina, T. Gustavsson and D. Carriere, Soft Matter, 2009, 5, 4212-4218.

23 E. Maurer, L. Belloni, T. Zemb and D. Carriere, Langmuir, 2007, 23, $6554-6560$.

24 D. Caspar and A. Klug, Cold Spring Harbor Symp. Quant. Biol., 1962, 27, 1-24.

25 E. Aarts and J. Korst, Simulated Annealing and Boltzmann Machines, John Wiley and Sons Inc, New York, 1988.

26 H. S. Seung and D. R. Nelson, Phys. Rev. A: At., Mol., Opt. Phys., 1988, 38, 1005-1018.

27 T. C. Lubensky and J. Prost, J. Phys. II, 1992, 2, 371-382.

28 C. M. Ghim and J. M. Park, J. Phys.: Condens. Matter, 2003, 15, 3891-3907.

29 K. R. Mecke, T. Charitat and F. Graner, Langmuir, 2003, 19, 20802087.

30 C. H. Lee, W. C. Lin and J. P. Wang, Phys. Rev. E: Stat., Nonlinear, Soft Matter Phys., 2001, 64, 020901.

31 E. Evans and W. Rawicz, Phys. Rev. Lett., 1990, 64, 2094-2097.

32 G. Vernizzi and M. O. de la Cruz, Proc. Natl. Acad. Sci. U. S. A., 2007, 104, 18382-18386.

33 M. Dubois, V. Lizunov, A. Meister, T. Gulik-Krzywicki, J. M. Verbavatz, E. Perez, J. Zimmerberg and T. Zemb, Proc. Natl. Acad. Sci. U. S. A., 2004, 101, 15082-15087.

34 D. Carriere, L. Belloni, B. Deme, M. Dubois, C. Vautrin, A. Meister and T. Zemb, Soft Matter, 2009, 5, 4983-4990.

35 Y. Michina, D. Carriere, T. Charpentier, R. Brito, E. Marques, J. Douliez and T. Zemb, J. Phys. Chem. B, 2010, 114, 1932-1938.

36 N. Delorme, M. Dubois, S. Garnier, A. Laschewsky, R. Weinkamer, T. Zemb and A. Fery, J. Phys. Chem. B, 2006, 110, 1752-1758.

37 M. Widom, J. Lidmar and D. R. Nelson, Phys. Rev. E: Stat., Nonlinear, Soft Matter Phys., 2007, 76, 031911.

38 D. Raoult, B. La Scola and R. Birtles, Clin. Infect. Dis., 2007, 45, 95 102. 